\title{
BMJ Open Singing for people with aphasia (SPA): a protocol for a pilot randomised controlled trial of a group singing intervention to improve well-being
}

Mark Tarrant, ${ }^{1}$ Mary Carter, ${ }^{1}$ Sarah Gerard Dean, ${ }^{1}$ Rod S Taylor, ${ }^{1}$ Fiona C Warren, ${ }^{1}$ Anne Spencer, ${ }^{1}$ Jane Adamson, ${ }^{1}$ Paolo Landa, ${ }^{1}$ Chris Code, ${ }^{2}$ Raff Calitri ${ }^{1}$

To cite: Tarrant M, Carter M, Dean SG, et al. Singing for people with aphasia (SPA): a protocol for a pilot randomised controlled trial of a group singing intervention to improve well-being. BMJ Open 2018;8:e025167. doi:10.1136/ bmjopen-2018-025167

- Prepublication history for this paper is available online. To view these files, please visit the journal online (http://dx.doi. org/10.1136/bmjopen-2018025167).

Received 18 July 2018 Revised 27 July 2018 Accepted 3 August 2018
Check for updates

(C) Author(s) (or their employer(s)) 2018. Re-use permitted under CC BY-NC. No commercial re-use. See rights and permissions. Published by BMJ.

${ }^{1}$ Institute for Health Research, University of Exeter Medical

School \& PenCLAHRC, Exeter, UK

${ }^{2}$ Department of Psychology,

University of Exeter, Exeter, UK

Correspondence to

Dr Mark Tarrant;

m.tarrant@exeter.ac.uk

\section{ABSTRACT}

Introduction The singing for people with aphasia (SPA) intervention aims to improve quality of life and well-being for people with poststroke aphasia. A definitive randomised controlled trial $(\mathrm{RCT})$ is required to assess the clinical and cost effectiveness of SPA. The purpose of this pilot study is to assess the feasibility of such a definitive trial and inform its design.

Methods and analysis A two-group, assessor-blinded, randomised controlled external pilot trial with parallel mixed methods process evaluation and economic evaluation. Forty-eight participants discharged from clinical speech and language therapy will be individually randomised 1:1 to SPA (10 group sessions plus a resource booklet) or control (resource booklet only). Outcome assessment at baseline, 3 and 6 months postrandomisation include: ICEpop CAPability measure for adults, Stroke and Aphasia Quality of Life, EQ-5D$5 \mathrm{~L}$, modified Reintegration into Normal Living Index, Communication Outcome After Stroke, Very Short Version of the Minnesota Aphasia Test, Service Receipt Inventory and Care Related Quality of Life. Feasibility, acceptability and process outcomes include recruitment and retention rates, with measurement burden and trial experiences being explored in qualitative interviews (15 participants, 2 music facilitators and 2 music champions). Analyses include: descriptive statistics, with 95\% Cls where appropriate; qualitative themes; intervention fidelity from videos and session checklists; rehearsal of health economic analysis.

Ethics and dissemination NHS National Research Ethics Service and the Health Research Authority confirmed approval in April 2017; recruitment commenced in June 2017. Outputs will include: pilot data to inform whether to proceed to a definitive RCT and support a funding application; finalised intervention manual for multicentre replication of SPA; presentations at conferences, public involvement events; internationally recognised peer reviewed journal publications, open access sources and media releases.

Trial registration number NCT03076736.

\section{INTRODUCTION}

Mortality rates following stroke are falling, but stroke remains a major cause of disability.
Strengths and limitations of this study

Built on a strong theoretical framework, this intervention takes an important step towards the exploration of improving psychosocial health for people living with poststroke aphasia.

- This study focuses on people with poststroke aphasia: an often neglected and under-represented patient population in research.

- This pilot randomised controlled trial study meets the MRC guidance on the development and evaluation of complex interventions and includes comprehensive patient and public involvement.

- This small scale study will be used to estimate between-group differences in outcomes, but does not have sufficient statistical power to make inferential between-group comparisons.

- The follow-up periods are relatively short compared with what would be necessary in a fully funded definitive trial.

About $33 \%$ of the 152000 people affected by a stroke each year in the UK have aphasia. ${ }^{1}$ Aphasia is a language disorder impacting on gesture, reading/writing, expression and comprehension of speech. In Devon, where we will conduct the proposed research, there are approximately 6000 people living with this condition. ${ }^{2}$

As well as impaired language functioning, people with aphasia often report poor psychosocial health, including reduced social participation, social isolation and lower levels of overall well-being-outcomes exacerbated by low levels of confidence and self-efficacy in social situations ${ }^{3}{ }^{4}$ - and also depression and distress. ${ }^{5}$ Stroke survivors without aphasia also experience ill-health psychosocial outcomes but they are substantially worse in people with aphasia. ${ }^{3}$ While speech and language therapy can improve functional communication of people with aphasia, ${ }^{6}$ there is a paucity of evidence for its impact on psychosocial 
outcomes: improvements in language do not appear to translate to significant improvements in well-being. ${ }^{7}$

Accordingly, guidelines recommend that rehabilitation should integrate people with aphasia back into the community and into social roles in order to improve psychosocial health. ${ }^{8}$ However, there is a notable lack of opportunities for people with aphasia in this regard; those available (eg, local support groups) often are aimed at all stroke survivors, which may not meet the specific psychosocial needs of people with aphasia. ${ }^{9}$ People with aphasia can lack the confidence to take up opportunities within the community which for others may seem more accessible. $^{10}$

Our project will pilot a new group singing intervention for improving psychosocial health in people with aphasia. The intervention is complex and integrates the established Information-Motivation-Behavioural (IMB) skills model of health behaviour change ${ }^{11}$ to support individuals in developing the social skills and confidence needed to improve psychosocial outcomes. There is a growing interest in the potential benefits of group interventions, including those focused on music making and singing, for those with long-term conditions. ${ }^{12-15}$ Social groups represent a valuable basis for self-definition, self-esteem and belonging and provide a 'lens' or social identity through which people make sense of their health. ${ }^{16-20}$ Groups provide support to members, known to be important to psychosocial functioning and adjustment, and they motivate behaviour and achievement of behavioural goals. ${ }^{1621}$ When people lose their connection to others (such as with aphasia), their capacity to interact successfully with the social world (and ultimately their health and well-being) is significantly compromised..$^{22}$ Providing opportunities for such people to join social groups offers a means of developing meaningful relationships with other people and reconnecting with society. ${ }^{23-26}$ Our project draws on and expands this literature following principles of intervention design outlined in the MRC framework. ${ }^{27}$

The core research question was put forward by a local stroke survivor who asked 'What are the benefits of singing for people with aphasia following stroke?' An initial scoping review revealed several case studies whose focus tended to be on impairment-based outcomes (with a debate as to whether singing in particular can impact on speech production). ${ }^{28-31}$ Notably, there were no randomised controlled trials (RCTs), either of groupbased or individual-based interventions, that targeted psychosocial outcomes of people with aphasia (or stroke more broadly) which would make the current project redundant. Some uncontrolled studies and studies employing qualitative methods suggested potential benefits of group singing on well-being for people with aphasia. ${ }^{12}$ These observations resonate with our own recent development study, in which 10 people with aphasia participated in the first session of the new intervention, ${ }^{32}$ and also discussions with people with aphasia who have offered enthusiastic support for a new group intervention that focuses on singing and targets more directly the psychosocial problems that they experience.

Many people with aphasia retain the ability to $\operatorname{sing}^{33}$ and are able to participate in music-making activities. Most people enjoy music and singing, and group singing is by definition social, demanding cooperation and coordination between participants. ${ }^{12} 34$ People with aphasia in our development study told us that being in a singing group, more so than other activities like arts and crafts, would help them to interact with other people. ${ }^{32}$ In short, by bringing people with aphasia together in a singing group, it may be possible to improve aspects of their life that are currently experienced as significant barriers to psychosocial functioning and which are not currently addressed through existing healthcare service provision.

\section{Aims}

Singing for people with aphasia (SPA) aims to improve psychosocial health in people with aphasia by providing interactive opportunities which promote confidence (eg, in forming new relationships), reduce social isolation and increase well-being. A definitive RCT is required to assess the effectiveness and cost-effectiveness of the SPA intervention. The purpose of this pilot study is to help us plan such a trial. The study will assess whether the intervention is feasible and acceptable to participants, focusing on (1) how well we can recruit and retain participants, (2) whether we can successfully collect outcome measures at different assessment points and (3) how well the intervention can be delivered in different locations with different groups. Finally, the study will allow us to document the intervention complexity and likely mechanisms of action.

\section{METHODS AND ANALYSIS Design}

A two-group, assessor-blinded, randomised controlled external pilot trial with parallel mixed methods process evaluation and economic evaluation. Eligible participants will be individually randomised 1:1 to SPA (10 week singing programme plus a resource booklet) or control (resource booklet only). The design is depicted in figure 1, which shows the flow of participants throughout the trial.

\section{Population}

People with aphasia are often excluded from research on the presumption that they are unable to provide consent. ${ }^{35}{ }^{36}$ Our intervention is an inclusive intervention which does not discriminate on the basis of aphasia severity, time since stroke or singing ability. Additional support to engage with the research process may be required for people with communication disability and cognitive impairment. The Mental Capacity $\mathrm{Act}^{37}$ will be used as a framework for assessing participants' ability to make an informed decision about engaging in the research. For those with cognitive impairment, we will follow NRES guidance on involving personal or nominee consultees to facilitate their involvement in research. 


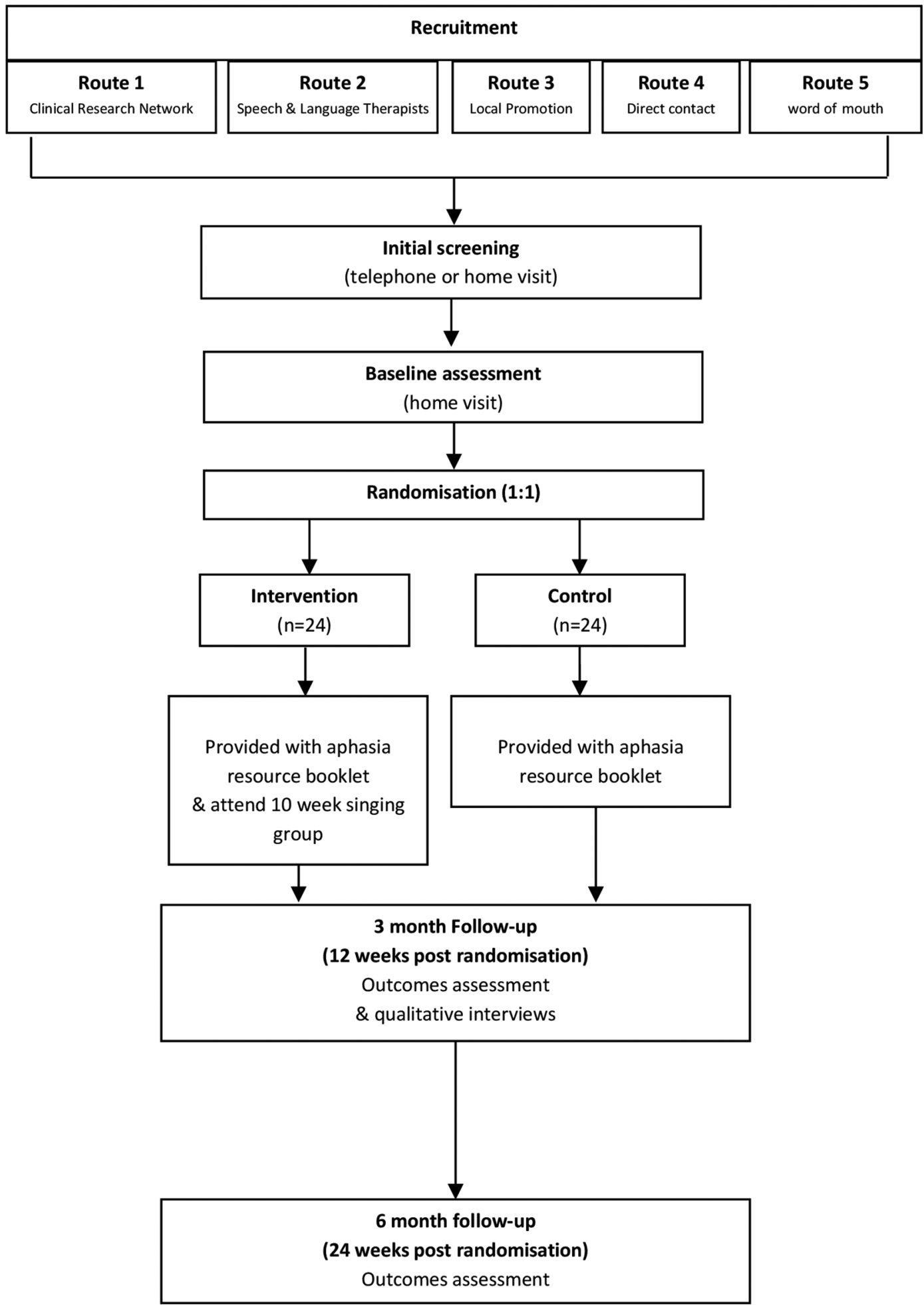

Figure 1 Study flowchart.

We will also use Accessible Information Guidance of the Stroke Association, and the National Institute of Health Research, and work with Speakability, our Service User Group (SUG) and a speech and language therapist (SLT) to ensure information is appropriately presented (eg, including use of pictures).

Potential participants will be included if they meet the following inclusion and exclusion criteria: they (1) have a diagnosis of aphasia after stroke (via referring clinician; the trial SLT via the Minnesota Aphasia Test; GP/medical records; self-report or carer-report), (2) are willing to be randomised to either control or SPA (and able to attend the singing venue), (3) had prestroke conversational English and (4) have capacity to consent. Potential participants will be excluded if they are: (1) less than 18 years old, (2) currently engaged in a SLT programme, (3) 
intending to relocate outside the geographical region, (4) participating in another study involving a lifestyle intervention or (5) currently attending a singing/music group.

\section{Sample size}

The target recruitment number is 48 participants (24 per arm). This number is based on the recommendation of 30 participants with complete data sets for pilot studies in order to estimate outcome variance ${ }^{38}$ and running the intervention three times to enable investigation of variations in context and other process variables. This recruited number is based on an estimate of the attrition rate across 6 months of $20 \%$ and allows estimation of this attrition with a precision of $\pm 13 \%$ with $95 \%$ certainty.

\section{Participant recruitment}

Participants will be recruited from Devon and surrounding areas, so participants will be local to the three programme delivery sites (Plymouth, South Devon and Exeter). These areas cover a population of 250000 with at least 3000 stroke survivors who require support with daily activities. ${ }^{2}$ To maximise potential involvement, several routes for recruitment will be used to reach both those who are just leaving SLT services and those who have been discharged for some time:

1. South West Peninsula Clinical Research Network (Stroke) will identify potential participants using:

- existing research databases,

- clinic lists,

- liaison with local clinicians.

2. SLTs will provide study information and patient information sheet (PIS) to potential participants:

- in person, at the end of their SLT programme,

- via post, following a database search and review of clinic list in order to contact previous SLT users.

3. Direct contact. The study will be advertised with:

- local support groups,

- on relevant websites (eg, The Stroke Association) and community newsletters.

4. Promotion via local stroke support networks identified through national organisations such as the Stroke Association, Different Strokes and via internet searches and in local media (radio and television).

5. Word of mouth, study flyers, adverts and information sheets.

For (1) and (2), potential participants will be given a PIS, including a brief description of the study and, if they are interested, permission will be obtained to pass their details to the research team. The participant will complete, with assistance if required, an expression of interest form, recording their contact details and consent for these details to be passed to the research team. Those contacted by post will be asked to return the expression of interest form by post direct to the research team. For (3), (4) and (5) above, potential participants will be able to self-refer by contacting the research team. Letters, articles and posters will be used to provide brief details of the study and invite expressions of interest to contact the research team by telephone call, email or post.

Because of uncertainty in recruitment rates and patterns and the need to have at least 12 people ready for randomisation, some participants may have to wait several weeks until sufficient group members have been consented. We estimate a delay of 4-8 weeks between consent and randomisation. Discussions with regional SLT services indicate that these predictions are realistic given the size of the local population of likely eligible participants. Once there are sufficient numbers, the Trial Manager (TM) will arrange a time/date to complete the baseline testing at each participant's home prior to randomisation. During the baseline assessment, the battery of questionnaires will be worked through with the participant.

Individuals will be informed of the potential delay to baseline assessment and randomisation during the initial screening phase. Those declining to participate at any stage of the recruitment process will be invited to share their reason(s) for declining, although will be under no obligation to give any.

\section{Randomisation and group allocation}

To ensure allocation concealment, participants will be allocated 1:1 to either intervention or control arms using a web-based randomisation service supported by the Exeter Clinical Trials Unit (ExeCTU). We will use a minimisation algorithm to ensure balance between groups, stratified by aphasia type (fluent vs non-fluent) and severity (mild/ moderate vs severe), gender and site (South Devon, Plymouth, Exeter). ExeCTU will perform each randomisation and inform the TM of group allocations; the TM will then relay group allocations to the participants.

\section{Blinding}

Participants, singing group facilitators providing the intervention and researchers conducting the process evaluation cannot be blinded to allocation. However, outcomes will be assessed by an independent assessor blinded to group allocation. Immediately prior to outcome assessments, participants will be reminded not to disclose their allocation to the assessor. Any incidents of unblinding will be recorded (including the stage at which it occurredprior to, during or postassessment), and the assessor will be asked to record their estimate of participant allocation prior to undertaking the assessments: we did this successfully in an earlier study. ${ }^{39}$ Following recommended strategies to maintain and assess blinding, ${ }^{40}$ the outcomes assessor will not be based at the research centre.

\section{Intervention}

SPA is based in a community centre/facility with 10 weekly singing group sessions. The intervention was developed with input from stroke survivors with aphasia, music facilitators, SLTs, health researchers and psychologists, following principles of participatory design. ${ }^{41} 42$ Development meetings covered structural (eg, location, facilities, seating arrangements, breaks), musical (eg, 
song book content and number of songs) and non-musical (eg, facilitator-led skills development) issues, including a consideration of potential barriers to and facilitators of participant engagement with the singing sessions. ${ }^{32}$

Each SPA session will last $90 \mathrm{~min}$, comprising $30 \mathrm{~min}$ of settling in/wrap-up and departure and $60 \mathrm{~min}$ of group singing. Sessions will be led by a community music leader, cofacilitated by a person with aphasia ('music champion'), and involve the group singing songs from a songbook. Song flow is aided by musical accompaniment (keyboard/guitar) provided by the facilitator(s). Small auxiliary percussion instruments (eg, shakers, tambourines) will be available for participants to play, enabling involvement of those with limited singing ability. Intervention content integrates the IMB skills model of health behaviour change ${ }^{11}$ to support individuals in developing the social skills and confidence needed to improve psychosocial outcomes. Providing people with information which they may lack (eg, about potential well-being benefits of participating in a singing group), bolstering change motivation (eg, providing positive feedback on participation) and teaching behavioural skills (eg, self-monitoring of behaviour and outcomes) are central to the IMB model and are also integral to our intervention. We draw on this framework to encourage formation of shared social identity among group participants from the outset of the singing programme and employ specific techniques from it to ensure its maintenance across the programme (eg, through the use of: an singing champion, inclusive language, group name and goal setting). Figure 2 describes the IMB skills components of each session. Sessions 2-9 will be flexible in terms of when each IMB skills component will be introduced, so as to accommodate individual progression within the group (the importance of which was emphasised in our earlier development study ${ }^{32}$ ); only a selection of nominated components will be used in any one session.

An Intervention Delivery manual will be developed and refined as part of the feasibility work of this study and will specify the essential resources, activities and behaviours of both facilitators and recipients that must be present in sessions and across the programme. Session check lists, completed by facilitators, will capture whether the main content of the Intervention Manual is being delivered, indicate where flexibility of delivery is permitted (in session structure/content) and allow evaluation of intervention fidelity. The trial SLT will contribute to training of music facilitators with a particular focus on overcoming potential barriers to participant communication.

\section{Control}

All participants (intervention and control) receive treatment as usual (which will be recorded) and a resource pack which collates information about living with aphasia and local community activities.
Assessment and outcomes

\section{Initial screening assessment}

Once a potential participant has been identified and has agreed to be contacted, the TM or Associate Research Fellow (ARF) will contact them to explain the study in full, answer questions and undertake eligibility screening. A family member, carer or guardian of the potential participant may speak with us on behalf of the participant. If a potential participant is unable to communicate over the phone and no carer or family member can answer eligibility questions on their behalf, we will visit them at home. Basic demographic data (eg, gender, date of birth, time since stroke) will also be collected during the exchange. For those who choose not to participate (or who are not eligible) at this stage, data will still be collected if the individual is happy to provide it. These data will allow description of the sample approached compared with the target population. After talking to a potential participant about the trial, eligible participants will be encouraged to think about taking part and to discuss the study with their family/friends/carers before making a decision. Anyone who is not eligible to take part will be thanked and informed that they are unable to participate. Potentially eligible participants will be asked to provide verbal consent for the study team to contact their GP to confirm the diagnosis of stroke and aphasia, if recorded on the patient records. This verbal consent will be documented by the research staff. One week later the TM or ARF will telephone, email or send a letter to the eligible participant to ask whether they would like to take part in the research study. If the person is agreeable, a home visit from the TM or ARF will be arranged and the TM or ARF will conduct further screening to confirm eligibility and will obtain written consent from eligible participants. Following this, full demographic and medical history data will be collected. If in the interim the GP report indicates an individual is not eligible for the trial, the study team will contact the individual and inform them that they are unable to participate. Any scheduled home visit will be cancelled.

\section{Home-based screening and data collection}

Those apparently eligible for inclusion will be visited at home by a member of the research team to provide further information about the study, confirm eligibility and to obtain written consent. Although potential participants will be asked at this time to give their consent for being video-recorded and photographed during singing sessions (should they be allocated to the intervention arm), and for taking part in an interview about their experiences at the end of the trial, these activities will also be specifically consented later in the trial process. Participants will be informed that video and photographic records are to allow the research team to retain a record of their activities and that we may present these images, with their permission, for academic or educational purposes. During the singing sessions, all participants will be asked to provide verbal consent immediately prior to 


\section{Information}

- $\quad$ Provide verbal or visual information that the facilitators are credible sources/experts in running the sessions.

- Provide information about the health/emotional consequences of joining the session: explain that singing and making music together can improve feelings of wellbeing.

- $\quad$ Provide information or facilitate discussion about what others think (like or approve) about the singing/music making.

- Instruct participants on how to perform the behaviour (e.g., use of instruments).

- Inform participants that there will be verbal reward (social incentives) if there has been effort to engage with the singing/music making.

- Problem solving to analyse factors influencing participant behaviours to overcome barriers/improve facilitation for subsequent sessions.

Motivation

- $\quad$ Set or explain that tasks/exercises will be graduated starting with easy, achievable tasks.

- $\quad$ Ask participants (and partners/carers) to support each other by providing practical or emotional support (e.g., helping with music equipment and offering praise).

- Downward comparisons: draw attention to participants' success during the session (improvements from earlier in the session or previous sessions).

- $\quad$ Prompt observation of the consequences of performing the behaviour (e.g., positive feedback for singing/music making).

- $\quad$ Prompt self/group-praise (social reward) when there has been effort to engage in singing/music making.

- Provide feedback on singing/music making behaviour (e.g., individual and collective improvement in singing quality).

- Ask participant to make a commitment to attending all the sessions using "I will" statements about returning to subsequent sessions.

- Provide feedback on behavioural outcomes (how much singing/music making may be improving wellbeing over the sessions).

- $\quad$ Goal setting (behaviours) and action planning (e.g., for the session).

\section{Behaviour}

- Promoting positive and valued social-identity: self-affirmation as a member of the singing group; use inclusive language ("us", "we") to reinforce new social identity as singing group member.

- $\quad$ Demonstrate the behaviour (e.g., facilitator performs singing/music making).

- $\quad$ Facilitate physical participation (e.g., breathing exercises for singing; making sounds).

- Identity framing/reframing: encourage participants to make links between their singing/music making and wellbeing (cognitive reframing).

- $\quad$ Prompt assessment and sharing of feelings after singing/music making and encourage participants to record or state their feelings after the session.

- $\quad$ Promote generalisation of behaviour (singing/music making), advising participants to sing or make music at home / with friends.

Figure 2 The IMB skills components of the SPA programme. IMB, Information-Motivation-Behavioural skills; SPA, singing for people with aphasia

video-recording or having their photograph taken so that they can confirm or change their original consent. They will also be asked whether they would like their face to be blurred out during any editing of the recordings. Carers and singing group facilitators attending group singing sessions or interviews will be taken through the same consenting procedures as the participants.

Demographic and other personal information collected at the consent visit comprise:

- Date of birth, gender, ethnicity.
- Stroke and aphasia history (Very Short Version of the Minnesota Aphasia Test, ${ }^{43}$ summary of speech and language therapy including length of programme, duration of sessions, confirmation of discharge). Participants' completion of the Very short Minnesota Aphasia Test will be audio recorded so that the trial SLT can categorise severity of aphasia. Audio recordings will be permanently deleted after categorisation.

- Modified Rankin Scale Score. ${ }^{44}$

- Medical history (comorbidities, current treatments). 
- Social history (employment status, previous employment, Index of Multiple Deprivation).

- Preference/need for carer presence in the intervention.

- Broad musical preferences (to inform planning of singing sessions).

Depending on recruitment patterns and due to the group format of intervention, eligible and consenting participants may have to wait several weeks before commencement of the intervention. They will be kept informed of timings by the TM using the participants' preferred method of communication (telephone, email or post).

\section{Clinical outcomes}

All clinical outcome measures will be collected for all participants during face-to-face assessments in their own home at baseline by the TM or ARF and at 3 and 6 months postrandomisation by a research practitioner blind to trial arm allocation. Measures include: (1) Wellbeing (ICECAP-A) ${ }^{45}$; (2) Stroke and Aphasia Quality of Life Scale (SAQOL) ${ }^{46}$; (3) EuroQol Quality of Life Scale (EQ-5D-5L) ${ }^{47}$; (4) Social participation (modified Reintegration to Normal Living (mRNL) Index) ${ }^{48}$; (5)
Communication (COAST) ${ }^{49}$; (6) Aphasia (Very Short Version of the Minnesota Aphasia Test) ${ }^{43}$ and care related quality of life (CarerQoL 7-D) ${ }^{50}$ for carers. Health and social service use will be measured through a Service Receipt Inventory. ${ }^{51}$ Adverse events will be reported for the week prior to the research visit. Table 1 indicates the outcome instruments and the time points for their administration.

\section{Feasibility, acceptability and process outcomes}

The feasibility of a definitive RCT will be determined by collecting and analysing the following pilot study data: (1) Numbers and details of those approached; (2) Recruitment and retention rates, including recruitment patterns from each route and geographical area. Those who decline to participate or who drop out of the study will be asked to provide their reasons for this; (3) Acceptability of randomisation; (4) outcome measurement burden; (5) interventions and other aspects of trial participation will be investigated by checking completion of questionnaires as well as through interviews with 15 participants (purposively selected to ensure inclusion of different genders, ages, time since stroke (under/over 1 year), severity of aphasia, past experience of singing,

Table 1 Trial outcome measures and when used (1=baseline; 2=3-month follow-up; 3=6-month follow-up)

\begin{tabular}{|c|c|c|c|c|}
\hline \multirow[b]{2}{*}{ Measure } & \multirow{2}{*}{$\begin{array}{l}\text { Time } \\
\text { (min) }\end{array}$} & \multicolumn{3}{|c|}{ Assessment } \\
\hline & & 1 & 2 & 3 \\
\hline \multicolumn{5}{|l|}{ ICEpop CAPability measure for Adults (ICECAP-A) ${ }^{45}$} \\
\hline
\end{tabular}

\section{Stroke and Aphasia Quality of Life Scale (SAQOL-39) ${ }^{46}$}

A 39-item health-related quality of life measure with four subdomains: physical, psychosocial, communication and energy.

EQ-5D-5L ${ }^{47}$
A 6-item measure of health-related quality of life that can be used for cost utility analysis.
modified Reintegration to Normal Living (mRNL)
$\begin{aligned} & \text { An 11-item measure that captures social participation (eg, recreation, movement in the community } \\ & \text { and interaction in family or other relationships). }\end{aligned}$

\section{Communication Outcome After STroke (COAST) $)^{49}$}

A 20-item measure of communication effectiveness for people with any type of communication problem following stroke

\section{Very Short Version of the Minnesota Aphasia test ${ }^{43}$}

A diagnostic tool to identify aphasia type. Comprised four activities: Identifying names, oral reading 15 words, naming pictures and written spelling. Audio-recorded to allow for diagnosis of aphasia severity.

\section{Service Receipt Inventory ${ }^{51}$}

Record of types and amount of use of health and social care resources including clinical contacts, 20 formal and informal social care. Completed by Assessor drawing on participant and family accounts.

Care related Quality of Life (CarerQoL-7D)
For completion by carers. A 7-item measures of the impact of providing informal care on carers.
Utility tariffs to calculate a weighted sum score of the CarerQol-7D are also available.
Adverse incidents ${ }^{52}$
\begin{tabular}{l}
$\checkmark$ \\
\hline
\end{tabular}


trial arm and cohort location) and by interviews with the singing group facilitators and singing champions. These qualitative interviews will use a semistructured interview schedule designed to cover the above issues and will ask about other personal and contextual factors that may affect participation and outcomes (ie, barriers and facilitators) including what refinements might be needed for the definitive trial delivery. Interviews will be conducted by a member of the research team at a convenient time and location for the participant after the intervention period; (6) Any adverse events ${ }^{52}$ will be identified, via singing group facilitator reports and the research staff explicitly questioning participants during the follow-up assessments, using trial standard operating procedures (no adverse events were reported in our development study); (7) Intervention fidelity will be assessed by several methods: singing group attendance, session checklists, observations and video recordings of selected singing group sessions in the programme (undertaken by the TM or ARF).

\section{Usual care}

Participants in both intervention and control arms may receive health and social care as part of their usual care, and these will be recorded using the Service Receipt Inventory.

\section{Foreseen difficulties}

(1) 'Singing for aphasia' may be seen by some as implying that the intervention is aimed specifically at improving language function of people with aphasia. Our SUG and development study participants strongly advised that we emphasise the well-being and quality of life benefits of SPA over its potential for impacting language function (there is very little supporting empirical evidence for the latter impact). Nevertheless, we will be assessing the impact of the singing group on speech and language. Our patientfacing materials reflect this. (2) Timing: as a group intervention, some of those who give consent to take part may have to wait several weeks until the SPA programme is ready to commence. The study team will maintain brief but regular contact with those waiting. The feasibility and acceptability of this process, including the wait time and recruitment/retention issues more generally, are part of what is being tested in this pilot study. (3) Burden (participant): particularly completion of the battery of measures and travel to intervention venue. These were deemed acceptable by most participants in our preliminary work. However, rather than asking participants to complete all questionnaires in one long session, we will offer to make multiple visits. To ensure we do not overburden participants, these visits will be restricted to no more than 1 hour (unless participants wish to continue beyond 1 hour) and will allow participants to take breaks as required. There may also be burden on carers: they may need to provide travel to and from the 10 weekly singing sessions at the community centre venues for participants in the intervention arm. (4) Facilitator availability: we will have secured the input of a singing facilitator through a community organisation in Plymouth and another facilitator, via local contacts, for the cohorts in South Devon and Exeter.

\section{Service user involvement}

Stroke survivors, their partners and carers have been consulted at all stages of the work leading to this protocol. Our patient and public involvement (PPI) representatives have materially influenced decisions on the study population, promotion and recruitment, the nature of the SPA intervention and how its effectiveness should be assessed. One experienced PPI representative will be part of the Trial Management Group (TMG), and another will join the Trial Steering Committee (TSC). A total of nine people have joined our SUG for this pilot study and will contribute by reviewing study materials and documentation, commenting on and proof reading reports and contributing to dissemination activities. This group has worked with us for several years already and will continue to be supported in their work by Peninsula Collaboration for Leadership in Applied Health Research and Care (PenCLAHRC) PPI team, for example, by attending workshops on critical appraisal skills.

\section{Data analysis}

Given the feasibility objectives of this pilot study, the focus of data analysis will be descriptive. For both recruitment settings (North, East and West (NEW) Devon and South Devon), participant progression through the study will be reported using a CONSORT flow diagram. We will report recruitment rates by site and levels of attrition at 3 and 6 months. The diagram will also reflect the number of recruitment letters sent, numbers consenting, number randomised, number undertaking intervention and number of completed outcomes alongside means and SD regarding the number, length and frequency of sessions. All protocol deviations, along with reasons and number of missing items on questionnaires will be reported. Descriptive statistics (mean and SD or equivalent) will be reported for demographic data and all outcome measures at each assessment point for both the intervention and control groups. A longer follow-up assessment point (eg, 12-24 months) needed for the definitive trial is beyond the scope of this pilot trial; however, we will use observed attrition at 3 and 6 months to model the potential level of attrition at longer follow-up.

For the trial process evaluation, we will use a thematic analysis for the qualitative interview data and use several of the quantitative measures (including demographic, medical, video and questionnaire data) to help identify and understand potential mediators and moderators of trial outcome. For the analysis of intervention fidelity and engagement we will use trainer interview data, session checklists completed by facilitators (that will be part of the Intervention Delivery Manual), participant attendance data, researcher observations and videos of singing sessions. These data collection and analysis methods will be assessed for their potential to inform 
Table 2 Study timeline

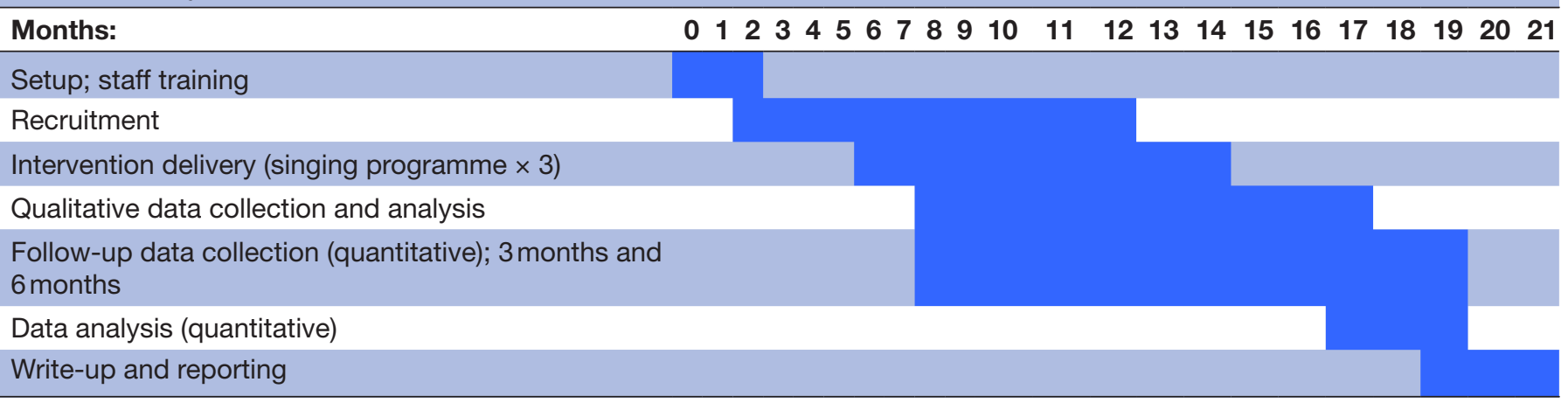

the process evaluation component of a definitive trial. In such a future trial, the health economics analysis will be a cost-utility analysis, using Quality Adjusted Life Years and a secondary analyses will investigate the benefits of the intervention more broadly within the framework of a cost consequences approach, so offering the potential to weight different outcomes in a multicriteria decision analysis framework. ${ }^{51}$ In this pilot study, we will assess costs associated with intervention delivery as well as assess the feasibility of collecting health and social service resource use through a Service Receipt Inventory.

\section{Study timeline}

The broad timetable for the research can be seen in table 2: 0-2 months: Staff training, study setup; 2-8 months: Participant recruitment; 6-11 months: Intervention delivery; 8-13months: Qualitative data collection and analysis; 8-17 months: Follow-up data collection (quantitative); 17-19 months: Data analysis; 19-21 months: Write-up, revise intervention manual, final report, dissemination. Trial management, steering committee and SUG meetings will be held on 14 occasions total across the 21 months of the study.

\section{Patient and public involvement}

PPI is central to the progression of the research study. The core research question was put forward by a stroke survivor who wanted to know what the benefits of singing were for people with poststroke aphasia. Our SUG reviewed questionnaires and supported their transformation into aphasia friendly format. They also advised on study venue and structure of the singing group sessions. Our SUG will be kept informed on the progress of the study via regular newsletters and a final 'dissemination' meeting will be held with them to present results and seek advice on reporting findings to study participants. We will send a summary of results to all study participants in aphasia friendly format. We will have independent patient representatives on the TSC and TMG. The TMG member will contribute to the review and editing of all academic outputs and will be an important coauthor.

\section{ETHICS AND DISSEMINATION \\ Ethics}

The study will be conducted in accordance with the principles of the International Conference for Harmonisation of Good Clinical Practice (ICH GCP) guidelines ${ }^{53}$ and the Research Governance Framework for Health and Social Care ${ }^{54}$ Any amendments to the trial documents will be approved by the sponsor before submission to the REC and HRA. The University's Clinical Trials insurance cover provides either legal liability cover or non-negligent/ no fault compensation cover.

We believe that the trial does not pose any specific risks to individual participants and nor does it raise any particular ethical issues. Based on results from our development work, SPA is a low $\square$ risk intervention. Individuals may report being distressed-either as a result of research activity or as a result of events in their private lives. Should such a situation arise, the researchers will implement the trial risk protocol and manage the participant in accordance with this policy. Under high-risk situations (eg, where there is perceived immediate risk to a participants health), the study team may be required to break confidentiality, to inform appropriate authorities who will need to provide essential care services. This information will be outlined in the Participant Information Sheet.

Participants will be informed of possible benefits and known risks of participation in the trial by means of a PIS and through discussion with the research team. All participants will sign a consent form approved by the ethics committee. They will be consented to participating in the trial, being randomised and followed up, participating in and being audio-recorded during interview, being video-recorded in the singing sessions and being contacted in the future about this and other research. Individuals who are not able or not willing to be randomised will not be recruited. Individuals will be sent an additional PIS relating to the interview study. Written consent will be obtained again immediately prior to the interview study; verbal consent will be obtained immediately prior to any video-recording of singing sessions: these consent processes will allow participants to fully consider their participation and to confirm or change their original consenting decision. 


\section{Governance and safety considerations}

Drafts of the trial protocol have been reviewed by the Southwest Research Design Service and the CRN. The trial is registered with the ClinicalTrials.gov: NCT03076736. This is a University of Exeter-sponsored research study, working in collaboration with NHS trusts.

\section{Adverse events}

As a non-physical intervention, there appears to be a low risk of serious adverse advents (AEs) associated with this study. Singing group facilitators will record any reported or witnessed AEs during the intervention and participants will be asked about AEs as part of the process evaluation (to maintain blinding of the outcomes assessor). All participants will also be asked to report on adverse events over the preceding week during their follow-up assessments. Any serious $\mathrm{AE}^{52}$ will be immediately reported to the trial sponsor and relevant ethics committee if the Chief Investigator deems it related to the intervention and to the independent TSC members who are also acting as our Data Monitoring and Ethics Committee.

\section{Trial monitoring and management}

Day-to-day running of the trial will be the responsibility of the TM. Standard Operating Procedures will be followed for: (1) assessment processes and reporting; (2) data management; (3) adverse incidents monitoring, reporting and action and (4) study staff health and safety.

The core study team (CI, TM and ARF) will meet weekly during the study. The TMG will include all of the core study team, all funding coapplicants and a person with aphasia. The TMG will review progress and provide advice on study planning. The TMG will meet on six occasions. TSC meetings will be held (if necessary by teleconference) on four occasions. The TSG will discuss recruitment, withdrawals, study progress, process evaluation and AEs and will advise on protocol amendments where necessary. The TSC will include academics with expertise in trial methodology, health economics, qualitative methods and process evaluation, a clinician with expertise in stroke and rehabilitation, a person with aphasia and a representative of the trial sponsor. Due to the low risk of AEs, an independent data monitoring and safety committee will not be appointed. Instead, we will appoint suitably qualified academics and clinicians to the TSC who will have responsibility for independently monitoring the safety and quality of the trial. A closed meeting prior to the TSC meetings will take place with the independent members of the TSC who will also be responsible for oversight of the safety of the trial and data integrity (thereby taking on the role of the data monitoring and ethics committee).

\section{Data management}

All study data will be kept for 10 years under secure conditions on University of Exeter secure servers. Data will also be subject to standard secure storage and usage policies. Data will be collected and retained in accordance with the
UK Data Protection Act 1998 and the new General Data Protection Regulation 2018 and managed in accordance with the trial-specific standard operating procedure for data management. With their consent, participant details will be passed between NHS services and the research team by telephone, post or in person only (that is, not electronically). All enrolled participants will be allocated a unique study ID, and the information linking their ID to their personal details will be kept securely at the University of Exeter. All other participant-related paper records will be anonymised and stored separately from the personal information. The electronic database for the trial will be stored on the secure servers of the University of Exeter with password-controlled access provided for the research team by the Exeter Clinical Trials Unit. Double data entry with extensive in-built validity checks will be used to reduce the risk of transcription errors. The study database will include prompts for missing data and warnings to alert staff when values are entered that are outside of the expected range or if the type of value entered is incorrect (eg, a numeric value entered rather than text).

Audio and video recordings will be digitised, encrypted and stored on the University's secure server. Audio recordings will be retained until after anonymised transcripts have been finalised and analysed. At this stage, they will be securely and permanently deleted. All participants (including carers and trainers) will provide verbal consent to video-recording on each occasion that it occurs. Everybody will be offered the option of having their image edited so they are not identifiable. Access to personal data will be restricted to the research team. Names and participant details will not be passed to any third parties and no named individuals will be included in the write-up of the results. All participants (people with aphasia, carers, singing group facilitators and music champions) will be asked for their consent for the study team to retain interview transcripts and video recordings for the purposes of future research by those involved directly in the study team or for educational purposes.

\section{Dissemination and impact activities}

Trial progress will be reported to our SUG at meetings and through quarterly newsletters. At the end of the study, we will seek input from our SUG to help disseminate a lay summary of the findings to study participants.

We envisage a number of key papers arising from this pilot trial. A trial publication policy will be developed which outlines the strategic plan for dissemination. The results of the trial will be reported first to study collaborators and to the funder (the Stroke Association). The main report will be drafted by the TMG and circulated to all collaborators and the TSC for comment.

Key outputs from the trial will contribute to our dissemination and impact agenda: (1) Pilot data will inform the decision whether to proceed to a definitive RCT and, if so, we will have (2) the evidence to support a funding application and (3) finalised Intervention Delivery manual to 
enable multicentre replication of SPA; (4) presentations at national and international conferences, seminars and PPI events and (5) dissemination through internationally recognised peer reviewed journal publications (including open access web sources), newsletters and media releases.

Acknowledgements We would like to thank the Stroke Association for funding this pilot RCT. We are very grateful for PenCLAHRC for their support of the study and to the Peninsula Patient Involvement Group for helping guide the trial design. We would also like to thank Plymouth Music Zone (PMZ) for their support of the study. PMZ was involved in the early development work including intervention design and they will provide resource (staff and facilities) to support the delivery of the intervention. We would also like to thank our SUG for their contributions to the design and development of the research study.

Contributors MT led the project team and the protocol development. RC, MT and $\mathrm{MC}$ wrote the first version of protocol and RC wrote all amendments for ethics submission. MT provided expertise on group processes in treatment settings. CC provided aphasia and speech and language expertise. RST provided statistical and trial methodological expertise. FCW provided statistical expertise and drafted the statistics data analysis plan. MC and SGD provided qualitative expertise and drafted the Health Economics analysis plan. SGD and MC provided process evaluation expertise. AS and PL provided health economic expertise. JA provided patient and public involvement expertise. RC and MC provided trial management expertise. All authors commented on the protocol and the manuscript.

Funding The trial is funded by the Stroke Association (QQ12 / TSA 2016/14). Excess treatment costs have been covered by South Devon and Torbay Clinical Commissioning Group, North East and West Devon Clinical Commissioning group, and the University of Exeter Medical School. This research was supported by the National Institute for Health Research (NIHR) Collaboration for Leadership in Applied Health Research and Care South West Peninsula at the Royal Devon and Exeter NHS Foundation Trust. We also acknowledge the support of the NIHR Clinical Research Network.

Disclaimer The views expressed are those of the author(s) and not necessarily those of the NHS, the NIHR or the Department of Health.

Competing interests All authors report the Stroke Association funding for the work under consideration but no other conflicts of interest.

Patient consent Not required.

Ethics approval Ethical approval for the study has been granted by theHealth Research Authority (HRA) and NHS National Research Ethics Service andResearch, via the Southwest - Frenchay Research Ethics Committee (17/SW/0060).

Provenance and peer review Not commissioned; peer reviewed for ethical and funding approval prior to submission.

Data sharing statement Not relevant at this stage.

Open access This is an open access article distributed in accordance with the Creative Commons Attribution Non Commercial (CC BY-NC 4.0) license, which permits others to distribute, remix, adapt, build upon this work non-commercially, and license their derivative works on different terms, provided the original work is properly cited, appropriate credit is given, any changes made indicated, and the use is non-commercial. See: http://creativecommons.org/licenses/by-nc/4.0/.

\section{REFERENCES}

1. The Stroke Association. State of the Nation Stroke Statistics 2016, 2017. https://www.stroke.org.uk/sites/default/files/stroke_statistics 2015.pdf. (accessed 16 Feb 2017).

2. Devon Wide Stroke Group. Health Equity Profile for Stroke, 2009.

3. Cruice M, Worrall L, Hickson L. Quantifying aphasic people's social lives in the context of non $\square$ aphasic peers. Aphasiology 2006;20:1210-25.

4. Dalemans RJ, De Witte LP, Beurskens AJ, et al. An investigation into the social participation of stroke survivors with aphasia. Disabil Rehabil 2010;32:1678-85.

5. Hilari K, Northcott S, Roy P, et al. Psychological distress after stroke and aphasia: the first six months. Clin Rehabil 2010;24:181-90.

6. Brady MC, Kelly H, Godwin J, et al. Speech and language therapy for aphasia following stroke. Cochrane Database Syst Rev 2012;16:Cd000425.
7. Franzén-Dahlin A, Karlsson MR, Mejhert M, et al. Quality of life in chronic disease: a comparison between patients with heart failure and patients with aphasia after stroke. J Clin Nurs 2010;19(1314):1855-60.

8. Dworzynski K, Ritchie G, Playford ED. Stroke rehabilitation: longterm rehabilitation after stroke. Clin Med 2015;15:461-4.

9. Howe TJ, Worrall LE, Hickson LMH. Interviews with people with aphasia: Environmental factors that influence their community participation. Aphasiology 2008;22:1092-120.

10. Le Dorze G, Salois-Bellerose Émilie, Alepins M, et al. A description of the personal and environmental determinants of participation several years post-stroke according to the views of people who have aphasia. Aphasiology 2014;28:421-39.

11. Fisher WA, Fisher JD, Harman J. The information-motivationbehavioral skills model: a general social psychological approach to understanding and promoting health behavior. Social Psychological Foundations of Health and IIIness: Blackwell Publishing Ltd 2009:82-106.

12. Tamplin J, Baker FA, Jones B, et al. 'Stroke a Chord': the effect of singing in a community choir on mood and social engagement for people living with aphasia following a stroke. NeuroRehabilitation 2013;32:929-41.

13. Clift SH, Staricoff G, Whitmore R. Singing and Health: A systematic mapping and review of non-clinical research: Sidney De Haan Research Centre for Arts and Health, 2008.

14. Skingley A, Clift SM, Coulton SP, et al. The effectiveness and costeffectiveness of a participative community singing programme as a health promotion initiative for older people: protocol for a randomised controlled trial. BMC Public Health 2011;11:142.

15. Clift SH G. The significance of choral singing for sustaining psychological wellbeing: findings from a survey of choristers in England, Australia and Germany. Music Performance Research 2010;3:79-96.

16. Haslam SA, Jetten J, Postmes T, et al. Social identity, health and well-being: an emerging agenda for applied psychology. Appl Psychol 2009;58:1-23.

17. Tarrant M, Hagger MS, Farrow CV. Promoting positive orientation towards health through social identity. The social cure: Psychology Press 2011:39-54.

18. Haslam C, Jetten J, Cruwys T, et al. The new psychology of health: Unlocking the social cure. Routledge, 2018.

19. Cameron JE, Voth J, Jaglal SB, et al. "In this together": Socia identification predicts health outcomes (via self-efficacy) in a chronic disease self-management program. Soc Sci Med 2018;208:172-9.

20. Tarrant M, Khan SS, Farrow CV, et al. Patient experiences of a bariatric group programme for managing obesity: a qualitative interview study. Br J Health Psychol 2017;22:77-93.

21. Oyserman D, Fryberg SA, Yoder N. Identity-based motivation and health. J Pers Soc Psychol 2007;93:1011-27.

22. Haslam C, Holme A, Haslam SA, et al. Maintaining group memberships: social identity continuity predicts well-being after stroke. Neuropsychol Rehabil 2008;18(5-6):671-91.

23. Haslam C, Haslam SA, Jetten J, et al. The social treatment: the benefits of group interventions in residential care settings. Psychol Aging 2010;25:157-67.

24. Knight C, Haslam SA, Haslam C. In home or at home? How collective decision making in a new care facility enhances social interaction and wellbeing amongst older adults. Ageing Soc 2010;30:1393-418.

25. Shadden BA JP. Renegotiation of identity: The social context of aphasia support groups. Topics in Language Disorders 2004;24:174-86.

26. Haslam C, Cruwys T, Haslam SA, et al. Groups 4 Health: Evidence that a social-identity intervention that builds and strengthens social group membership improves mental health. $J$ Affect Disord 2016;194:188-95.

27. Craig P, Dieppe P, Macintyre S, et al. Developing and evaluating complex interventions: the new Medical Research Council guidance. BMJ 2008;337:a1655.

28. Stuckey HL, Nobel J. The connection between art, healing, and public health: a review of current literature. Am J Public Health 2010;100:254-63.

29. Hurkmans J, de Bruijn M, Boonstra AM, et al. Music in the treatment of neurological language and speech disorders: A systematic review. Aphasiology 2012;26:1-19.

30. Stahl B, Kotz SA, Henseler I, et al. Rhythm in disguise: why singing may not hold the key to recovery from aphasia. Brain 2011;134:3083-93.

31. Tomaino CM. Recovery of fluent speech through a musician's use of prelearned song repertoire: a case study. Music Med 2010;2:85-8.

32. Tarrant M, Warmoth K, Code C, et al. Creating psychological connections between intervention recipients: development and focus 
group evaluation of a group singing session for people with aphasia. BMJ Open 2016;6:e009652.

33. Schlaug G, Norton A, Marchina S, et al. From singing to speaking: facilitating recovery from nonfluent aphasia. Future Neurol 2010;5:657-65

34. Pearce E, Launay J, Dunbar RI. The ice-breaker effect: singing mediates fast social bonding. $R$ Soc Open Sci 2015;2:150221.

35. Ali M, Bath PM, Lyden PD, et al. Representation of people with aphasia in randomized controlled trials of acute stroke interventions. Int J Stroke 2014;9:174-82.

36. Brady MC, Fredrick A, Williams B. People with aphasia: capacity to consent, research participation and intervention inequalities. Int $J$ Stroke 2013;8:193-6.

37. Gov.UK. Mental Capacity Act 2005. London: HMSO, 2005.

38. Browne RH. On the use of a pilot sample for sample size determination. Stat Med 1995;14:1933-40.

39. Dean SG, Poltawski L, Forster A, et al. Community-based rehabilitation training after stroke: results of a pilot randomised controlled trial (ReTrain) investigating acceptability and feasibility. BMJ Open 2018;8:e018409.

40. Siemonsma PC, Walker MF. Practical guidelines for independent assessment in randomized controlled trials (RCTs) of rehabilitation. Clin Rehabil 1997:11:273-9.

41. Clemensen J, Larsen SB, Kyng M, et al. Participatory design in health sciences: Using cooperative experimental methods in developing health services and computer technology. Qual Health Res 2007;17:122-30.

42. Wherton J, Sugarhood P, Procter R, et al. Co-production in practice: how people with assisted living needs can help design and evolve technologies and services. Implement Sci 2015;10:75.

43. Powell GE, Bailey S, Clark E. A very short version of the minnesota aphasia test. Br J Soc Clin Psychol 1980;19:189-94.
44. Bruno A, Akinwuntan AE, Lin C, et al. Simplified modified rankin scale questionnaire: reproducibility over the telephone and validation with quality of life. Stroke 2011;42:2276-9.

45. Al-Janabi H, Flynn TN, Coast J. Development of a self-report measure of capability wellbeing for adults: the ICECAP-A. Qual Life Res 2012;21:167-76.

46. Hilari K, Byng S, Lamping DL, et al. Stroke and Aphasia Quality of Life Scale-39 (SAQOL-39): evaluation of acceptability, reliability, and validity. Stroke 2003;34:1944-50.

47. Herdman M, Gudex C, Lloyd A, et al. Development and preliminary testing of the new five-level version of EQ-5D (EQ-5D-5L). Qual Life Res 2011;20:1727-36.

48. Miller A, Clemson L, Lannin N. Measurement properties of a modified Reintegration to Normal Living Index in a community-dwelling adult rehabilitation population. Disabil Rehabil 2011;33(21-22):1968-78.

49. Long A, Hesketh A, Paszek G, et al. Development of a reliable self-report outcome measure for pragmatic trials of communication therapy following stroke: the communication outcome after stroke (COAST) scale. Clin Rehabil 2008;22:1083-94.

50. Brouwer WB, van Exel NJ, van Gorp B, et al. The CarerQol instrument: a new instrument to measure care-related quality of life of informal caregivers for use in economic evaluations. Qual Life Res 2006:15:1005-21.

51. Craig LE, Wu O, Bernhardt J, et al. Approaches to economic evaluations of stroke rehabilitation. Int J Stroke 2014;9:88-100.

52. Carnes D, Mullinger B, Underwood M. Defining adverse events in manual therapies: a modified Delphi consensus study. Man Ther 2010;15.

53. Agency EM. Guideline for good clinical practice E6(R2. London: European Medicines Agency, 2016.

54. Department of Health. Research Governance Framework for Health and Social Care. 2 edn. London, 2005. 